\title{
Syncope: A Very Atypical Presentation of COVID-19
}

\author{
Richard D. Shih, Paul M. Louis, Scott M. Alter, Patrick G. Hughes, \\ Joshua J. Solano, and Lisa M. Clayton
}

\section{ABSTRACT}

Possible COVID-19 patients have presented commonly to United States Emergency Departments. Patients presenting with cough, fever and shortness of breath are easy to identify as suspected cases. Unfortunately, not all COVID-19 patients present this way. Atypical presentations are a risk factor for healthcare worker (HCW) transmission of this disease. In this case report we describe the unusual case of COVID-19 presenting as syncope without any other risk factors. HCWs need to be aware of atypical COVID-19 presentations and to maintain proper use of personal protective equipment.

Keywords: COVID-19, pandemic, syncope, healthcare worker transmission, viral pandemic.

Published Online: November 13, 2020

ISSN: $2593-8339$

DOI: $10.24018 /$ ejmed.2020.2.6.547

Richard D. Shih*

Florida Atlantic University Charles E.

Schmidt College of Medicine, Delray

Medical Cente, USA.

(e-mail: shih100@yahoo.com)

Paul M. Louis

Bethesda Hospital East, USA.

Scott M. Alter

Florida Atlantic University Charles E.

Schmidt College of Medicine, Delray

Medical Center, USA.

Patrick G. Hughes

Florida Atlantic University Charles E. Schmidt College of Medicine, Bethesda Hospital East, USA.

Joshua J. Solano

Florida Atlantic University Charles E.

Schmidt College of Medicine, Delray

Medical Center, USA.

Lisa M. Clayton

Florida Atlantic University Charles E. Schmidt College of Medicine, Bethesda Hospital East, USA.

*Corresponding Author

\section{INTRODUCTION}

Coronavirus disease 2019 (COVID-19) has overwhelmed many emergency departments during the current pandemic, and unfortunately transmission to healthcare workers is not uncommon [1]. Risk factors include unsuspected and atypical patient presentation, improper personal protective equipment (PPE) availability and technique, performance of high-risk procedures, and poor hand washing hygiene [2].

During this pandemic, all patients presenting to the emergency department have the potential to be infected with the disease. Atypical cases are particularly challenging to identify early on, and syncope in particular is an unusual presentation for COVID-19. Therefore, clinicians must maintain a high degree of suspicion for atypical COVID-19 presentations and use proper PPE for all patient contact during this pandemic. Our case involves a middle-aged male presenting after an acute syncopal event without any typical COVID-19 symptoms or risk factors, who was subsequently found to have bilateral pneumonia from COVID-19.

\section{CASE REPORT}

A 46-year-old previously healthy male was brought to the emergency department (ED) by paramedics after an episode of syncope and urinary incontinence. The patient is a construction worker and was at work when he suddenly clutched his chest and collapsed. Bystanders witnessing the event reported no tonic-clonic movements and no observation of illness prior to the event. No further information or past medical history was obtained at that time. On ED arrival, the patient was lethargic, breathing spontaneously, and reactive to painful stimuli only. His initial vital signs were blood pressure, $100 / 79 \mathrm{~mm} / \mathrm{Hg}$; heart rate, 120 beats per minute; respiratory rate, 28 breaths per minute; oral temperature, $97.2^{\circ} \mathrm{F}$; and pulse oximetry, $100 \%$ on room air. Point of care glucose was normal.

On physical exam, his pants were soiled consistent with urinary incontinence. Pupils were $4 \mathrm{~mm}$ and reactive bilaterally. There were no signs of head trauma. The oral mucosa was dry, the neck was supple, and there were no lacerations of the tongue. The lungs were clear to 
auscultation and the heart was tachycardic with a regular rhythm. The abdominal exam was unremarkable. Extremities were unremarkable with no signs of deformity, swelling, edema, tenderness, or palpable cords. The neurologic exam was non focal.

Initial ED assessment was consistent with syncope, altered mental status, and urinary incontinence. The patient was administered intravenous (IV) fluids as well as levetiracetam $500 \mathrm{mg}$ IV due to the possibility of seizure as the etiology of the patient's symptoms.

Initial radiography, including head CT and chest x-ray, were unremarkable. The electrocardiogram was normal aside from sinus tachycardia. Laboratory tests were significant for a white blood cell count of 11.7 thousand cells/uL with $28 \%$ lymphocytes, glucose of $167 \mathrm{mg} / \mathrm{dL}$, creatinine of $1.39 \mathrm{mg} / \mathrm{dL}$, troponin of $0.151 \mathrm{mg} / \mathrm{mL}$, and a negative urine drug screen.

Over the next 60 minutes, the patient's mental status returned to normal. Additional history was now obtained. The patient had been having a cough and subjective fevers over the past 5 days. In addition, he had no significant past medical history. He did not smoke cigarettes and had no allergies.

Vital signs repeated 1.5 hours later revealed the patient to now be febrile. Therefore, a sepsis work-up was initiated, including a nasopharengeal sample for COVID-19 testing. A non-contrast chest $\mathrm{CT}$ revealed acute bilateral mild pneumonia. The patient was treated with doxycycline 100 mg IV for coverage of pneumonia and admitted to the hospital telemetry unit. Deep vein thrombosis (DVT) prophylaxis was not administered.

On the day following admission, his condition was unchanged. Myocardial infarction was ruled out by trending of the troponin. COVID PCR test was positive, and he was administered hydroxychloroquine (400 mg orally, twice daily) and azithromycin.

After another 48 hours with no change in condition, on hospital day \#3, the patient suddenly developed shortness of breath, tachypnea, and hypoxia consistent with acute respiratory failure. He was emergently intubated and transferred to the ICU.

Doppler ultrasound of the lower extremities revealed a large DVT involving his left calf and thigh. Given acute hypoxia in the presence of DVT, the patient was presumed to also have a pulmonary embolus, and no confirmatory radiologic test was performed. The patient was anticoagulated with enoxaparin and his condition improved over the next several days. He was extubated on hospital day \#5 and discharged from the hospital on day \#8 on outpatient riveroxaban anticoagulation.

\section{DISCUSSION}

COVID-19 has infected millions worldwide and accounted for several hundred thousand deaths. EDs have been seeing a large number of suspicious presentations.

The symptoms of COVID-19 are well described [3]. In a United States study of COVID-19 cases, Garg, et. al found the most common presenting symptoms to be cough, fever or chills, and shortness breath.3 Each symptom occurred in
$80 \%$ or more of confirmed patients. Altered mental status or confusion was uncommon $(6.1 \%)$, and no cases of syncope or seizures were reported. In addition, $74 \%$ of cases involved patients older than 50 years [3].

In our case, the patient was 46 years old and presented to the ED with what witnesses described as sudden syncope with urinary incontinence. Seizure disorder was considered, although there was no history of tonic-clonic activity. Although the patient was initially afebrile and did not appear to have any pulmonary symptoms, fever developed 1.5 hours later, and nasopharyngeal testing confirmed COVID19.

When his condition worsened 2 days later, thromboembolic disease of the lower extremity was discovered via Doppler ultrasound. Although a chest CT angiogram was not obtained to confirm pulmonary embolus, this diagnosis was made presumptively given the sudden development of shortness of breath, tachypnea and hypoxia, along with the presenting symptom of acute syncope possibly resulting from venous thromboembolism (VTE).

VTE has been shown to be a common feature of critically ill COVID-19 patients, as approximately $25 \%$ will develop VTE or pulmonary embolus [4]. The basis for the increased risk of VTE in COVID-19 is unclear but has been theorized to be secondary to a virus-induced hypercoagulable state, reactive thrombocytosis, or cytokine-mediated diffuse microvascular damage [5].

Although most cases of COVID-19-induced pulmonary embolus present with shortness of breath, our patient's presentation with syncope is very unusual. Atypical COVID-19 presentations are one of the main risk factors for unsuspected viral transmission to healthcare workers (HCW) [2]. Risk factors for HCWs with respect to the acquisition of COVID-19 disease include unsuspected and atypical presentations in patients, poor PPE training and use, male gender, performance of high-risk procedures, and poor hand washing hygiene [2].

Although our case is a very atypical presentation of COVID-19, HCWs need to consider almost every ED patient as a suspected COVID-19 case. Maintenance of a high degree of vigilance, proper PPE use, and appropriate hand washing must be followed in all cases.

We present a case of acute syncope in an afebrile patient subsequently diagnosed with COVID-19, likely precipitating his cascade of illness. This atypical presentation exemplifies a major risk factor for potential transmission to unsuspecting healthcare workers. During current pandemic, all patients must be suspected of having COVID-19, proper use of personal protective equipment, and appropriate hand washing hygiene is essential in preventing healthcare worker COVID-19 disease.

\section{REFERENCES}

[1] CDC COVID-19 Response Team: Characteristics of health care personnel with COVID-19 - United States, February 12-April 9, 2020 MMWR Morb Mortal Wkly Rep. 2020; 69:477-481.

[2] Ran L, Chen X, Wang Y, et al. Risk factors of healthcare workers with corona virus disease 2019: A retrospective cohort study in a designated hospital of Wuhan in China. Clin Infect Dis. 2020 Mar 17. pii: ciaa287. doi: 10.1093/cid/ciaa287. 
[3] Garg S, Kim L, Whitaker M, et al. Hospitalization rates and characteristics of patients hospitalized with laboratory-confirmed coronavirus disease 2019 - COVID-NET, 14 states, March 1-30, 2020. MMWR Morb Mortal Wkly Rep. 2020; 69:458-464.

[4] Klok FA, Kruip MJHA, van der Meer NJM, et al. Incidence of thrombotic complications in critically ill ICU patients with COVID19. Thromb Res. 2020 Apr 10. pii: S0049-3848(20)30120-1. doi: 10.1016/j.thromres.2020.04.013. [Epub ahead of print].

[5] Mei H, Hu Y. Characteristics, causes, diagnosis and treatment of coagulation dysfunction in patients with COVID-19. Zhonghua xue ye xue za zhi Zhonghua xueyexue zazhi. 2020 Mar 5; 41: E002. 